\title{
Holographic Lovelock gravities and black holes
}

\author{
Jan de Boer, ${ }^{a}$ Manuela Kulaxizi ${ }^{a}$ and Andrei Parnachev ${ }^{b}$ \\ ${ }^{a}$ Department of Physics, University of Amsterdam, \\ Valckenierstraat 65, 1018XE Amsterdam, The Netherlands \\ ${ }^{b}$ C.N.Yang Institute for Theoretical Physics, Stony Brook University, \\ Stony Brook, NY 11794-3840, U.S.A. \\ E-mail: J.deBoer@uva.nl, M.Kulaxizi@uva.nl, parnachev@gmail.com
}

ABSTRACT: We study holographic implications of Lovelock gravities in AdS spacetimes. For a generic Lovelock gravity in arbitrary spacetime dimensions we formulate the existence condition of asymptotically AdS black holes. We consider small fluctuations around these black holes and determine the constraint on Lovelock parameters by demanding causality of the boundary theory. For the case of cubic Lovelock gravity in seven spacetime dimensions we compute the holographic Weyl anomaly and determine the three point functions of the stress energy tensor in the boundary CFT. Remarkably, these correlators happen to satisfy the same relation as the one imposed by supersymmetry. We then compute the energy flux; requiring it to be positive is shown to be completely equivalent to requiring causality of the finite temperature CFT dual to the black hole. These constraints are not stringent enough to place any positive lower bound on the value of viscosity. Finally, we conjecture an expression for the energy flux valid for any Lovelock theory in arbitrary dimensions.

Keywords: AdS-CFT Correspondence, Field Theories in Higher Dimensions, Black Holes, Anomalies in Field and String Theories

ARXIV EPRINT: 0912.1877 


\section{Contents}

1 Introduction and summary $\quad 1$

2 Lovelock gravity, black holes and fluctuations 3

$\begin{array}{lll}3 & \text { Black holes of 3rd order Lovelock gravity } & \mathbf{7}\end{array}$

3.1 Existence of black hole solutions 8

$\begin{array}{lll}3.2 & \text { Explicit solutions } & 9\end{array}$

4 Weyl anomaly and the correspondence between flux positivity and causal$\begin{array}{ll}\text { ity } & 16\end{array}$

$\begin{array}{llr}5 & \text { Discussion } & 19\end{array}$

A The viscosity/entropy ratio of the finite temperature CFT dual to Lovelock gravity.

\section{Introduction and summary}

Lovelock gravity [1] is the most general classical theory of gravity whose equations of motion contain at most second derivatives of the metric. The simplest example of Lovelock gravity is just the usual Einstein-Hilbert gravity. As the number of spacetime dimensions grows, Lovelock gravity allows more and more higher derivative terms. For example, in five dimensions one can add a term quadratic in the Riemann tensor, which gives rise to Gauss-Bonnet gravity. Important features of Lovelock gravity are the absence of ghosts in Minkowski backgrounds [2, 3] and the equivalence between metric and Palatini formulations [4]. One may wonder what role these higher derivative gravity theories play in the AdS/CFT correspondence [5-7]. The first interesting nontrivial example is Gauss-Bonnet gravity in an $A d S_{5}$ background which defines a four dimensional CFT. One can compute Weyl anomalies for this theory and find that the ratio of the two central charges, $a$ and $c$, depends nontrivially on the value of the Gauss-Bonnet coupling $\lambda_{1}$, and goes to one in the limit of vanishing $\lambda_{1}$ which corresponds to Einstein-Hilbert gravity [8-10]. Plenty of CFTs with $a \neq c$ are known, and having a gravity dual of such CFTs would be desirable.

In addition to the special features of Lovelock theories mentioned above, Gauss-Bonnet theory has been shown to have a peculiar property in the context of the AdS/CFT correspondence. Consider an $A d S_{5}$ solution of Gauss-Bonnet gravity which defines a dual four-dimensional CFT. Requiring positivity of the energy flux in a four-dimensional CFT places certain constraints on the values of the two parameters which determine the angular distribution of the energy flux, $t_{2}$ and $t_{4}$ [11]. Assuming supersymmetry, these constraints 
can be reformulated as bounds on the ratio of $a$ and $c$ central charges of the CFT, which in turn imply bounds on the value of Gauss-Bonnet coupling, $\lambda_{1}$. Exactly the same bounds on $\lambda_{1}$ have been obtained by requiring causality of the finite temperature CFT $[9,10,12,13]$. The story repeats itself for the six-dimensional CFT dual to Gauss-Bonnet gravity in $A d S_{7}$ [14]. In ref. [14] we computed the values of $t_{2}$ and $t_{4}$ in terms of three independent coefficients which determine the three and two point functions of the stress energy tensor. We observed that $t_{4}$ is proportional to the combination of the three point functions which vanishes in the supersymmetric theory. We computed the holographic Weyl anomaly for Gauss-Bonnet gravity and found that $t_{4}=0$ while the positivity of energy flux condition constrains the value of the Gauss-Bonnet coupling $\lambda_{1}$ to lie within a certain interval. We have also analyzed the propagation of a graviton with helicity 2 in the black hole background and found that the bound on $\lambda_{1}$ coming from causality precisely matches the lower bound on $\lambda_{1}$ from flux positivity. Generalizing the matching between causality and positivity of the energy flux to other polarizations in GB theories of arbitrary dimensions was achieved in $[15,16]$. In particular, [16] computed the three point functions of the stress-energy tensor and determined $t_{2}$ and $t_{4}$ in the CFTs dual to GB gravities in any dimensions.

In this paper we study Lovelock theories with negative cosmological constant, paying special attention to cubic Lovelock gravity in an $A d S_{7}$ background. In this case there are two independent coefficients which multiply the Gauss-Bonnet term and the term cubic in the Riemann tensor. In general the $\mathcal{O}\left(R^{3}\right)$ term is expected to describe a generic nonsupersymmetric case. However the third order Lovelock term does not contribute to the three-point functions of gravitons in flat space [17]. Yet, we show that the corresponding contribution in the AdS background does not vanish and hence the positivity of energy flux condition results in nontrivial constraints on both the Gauss-Bonnet and third order Lovelock coefficients, $\lambda_{1}$ and $\lambda_{2}$.

To find the constraints imposed by causality of the boundary theory we analyze the space of black hole solutions. We are able to formulate the conditions of black hole existence and compute the causality constraint for the graviton of helicity 2 in Lovelock gravity of arbitrary dimensionality. In the case of cubic Lovelock in seven spacetime dimensions we write down the black hole solutions explicitly; interestingly, for some values of the Lovelock parameters there are AdS solutions but no asymptotically AdS black holes. Remarkably, we find that this solution structure ensures that the causality constraint coincides with the energy positivity constraint. Another remarkable feature of Lovelock gravity is vanishing $t_{4}$, one of the parameters that determine the angular distribution of the energy flux. As explained in [14], $t_{4}$ is proportional to the linear combination of parameters which vanishes when the minimal supersymmetry is assumed. Constraints from flux positivity, together with the condition for the black hole to exist, restrict the value of the Gauss-Bonnet coupling from above. Interestingly, this restriction is not sufficient to place a positive lower bound on the viscosity/entropy ratio.

The rest of the paper is organized as follows. In the next section we recall the description of the most generic Lovelock gravity in any spacetime dimensions and formulate the conditions for the asymptotically AdS back holes to exist. We also analyze the propagation 
of gravitons of helicity 2 in these black hole backgrounds and derive bounds on the Lovelock coefficients resulting from causality. In section 3 we apply these results to the case of cubic Lovelock theory in seven spacetime dimensions. In particular, we write down explicit formulas for the black hole solutions. To the best of our knowledge, some of these solutions have not appeared in the literature before. In section 4 we compute the holographic Weyl anomaly in cubic Lovelock gravity in seven dimensions and determine the bounds on the Lovelock coefficients from flux positivity. We find that there is precise matching between the causality and positivity of flux bounds, just like in the Gauss-Bonnet case. We discuss our results in section 5 where, among other things, we discuss implications of our results to the viscosity/entropy ratio and make an educated guess for the energy flux in any Lovelock theory in arbitrary spacetime dimensions.

\section{Lovelock gravity, black holes and fluctuations}

Lovelock gravity [1] is the most general classical theory of gravity which yields covariant second order field equations, i.e., the equations of motion which contain only up to second order derivatives of the metric tensor. The Lovelock action for a $d+1$-dimensional spacetime is

$$
S=\int d^{d+1} x \sqrt{-g} \sum_{p=0}^{\left[\frac{d}{2}\right]} \beta_{p} \mathcal{L}_{p}
$$

where $\left[\frac{d}{2}\right]$ denotes the integral part of $\frac{d}{2}, \beta_{p}$ is the $p$-th order Lovelock coefficient and $\mathcal{L}_{p}$ defined as

$$
\mathcal{L}_{p}=\frac{1}{2^{p}} \delta_{\rho_{1} \sigma_{1} \cdots \rho_{p} \sigma_{p}}^{\mu_{1} \nu_{1} \cdots \mu_{p} \nu_{p}} R_{\mu_{1} \nu_{1}}^{\rho_{1} \sigma_{1}}{ }_{\mu_{p} \nu_{p}}^{\rho_{p} \sigma_{p}}
$$

is the Euler density of a $2 p$-dimensional manifold. Here $\delta_{\rho_{1} \sigma_{1} \cdots \rho_{p} \sigma_{p}}^{\mu_{1} \nu_{1} \cdots \mu_{p} \nu_{p}}$ denotes the totally antisymmetric product of Kronecker delta symbols while $R^{\rho_{q} \sigma_{q}}{ }_{\mu_{q} \nu_{q}}$ is the Riemann curvature tensor. By construction it is clear that in $d+1$ dimensions all Lovelock $\mathcal{L}_{p}$ terms for which $p \geq\left[\frac{d}{2}\right]$ either vanish (for $p>d / 2$ ) or are total derivatives (for $p=d / 2$ ) and do not contribute to the equations of motions. The $p=0$ and $p=1$ terms correspond to the cosmological constant and Ricci scalar respectively. Our conventions are such that $L=1$ while $\beta_{0}=d(d-1)$ and $\beta_{1}=1$.

To determine the black brane solutions of Lovelock gravity we consider the metric

$$
d s^{2}=-f(r) d t^{2}+\frac{d r^{2}}{f(r)}+r^{2} \sum_{i=1}^{d-1} d x_{i}^{2} .
$$

For this ansatz, the only non-vanishing components of the Riemann tensor (up to symmetries) are

$$
R_{t r}^{t r}=-f^{\prime \prime} / 2, \quad R^{t i}{ }_{t i}=R^{r i}{ }_{r i}=-\frac{f^{\prime}}{2 r}, \quad R^{i j}{ }_{i j}=-\frac{f}{r^{2}} .
$$

where primes indicate differentiation with respect to the variable $r$. This makes it relatively straightforward to evaluate the Lovelock action on a black hole solution of the form (2.3). 
The $p$-th Lovelock term evaluates as

$$
\int d^{d+1} x \sqrt{-g} \mathcal{L}_{p}=(2 p) ! ! \int d r d t d x^{d-1} r^{d-1}\left(Q_{1}+Q_{2}+Q_{3}+Q_{4}\right)
$$

where

$$
\begin{aligned}
& Q_{1}=-\frac{f^{\prime \prime}}{2} C_{p-1}^{d-1}\left(\frac{-f}{r^{2}}\right)^{p-1} \\
& Q_{2}=(d-1)(d-2)\left(\frac{-f^{\prime}}{2 r}\right)^{2} C_{p-2}^{d-3}\left(\frac{-f}{r^{2}}\right)^{p-2} \\
& Q_{3}=-\frac{f^{\prime}}{r}(d-1) C_{p-1}^{d-2}\left(\frac{-f}{r^{2}}\right)^{p-1} \\
& Q_{4}=C_{p}^{d-1}\left(\frac{-f}{r^{2}}\right)^{p}
\end{aligned}
$$

and $C_{p}^{d}$ is the number of ways you can pick $p$ pairs from $d$ numbers, i.e. $C_{p}^{d}=d ! /((d-$ $2 p) !(2 p) ! !)$. After some algebra, we find

$$
\int d^{d+1} x \sqrt{-g} \mathcal{L}_{p}=(-1)^{p} \frac{(d-1) !}{(d-2 p+1) !} \int d r d t d x^{d-1} \partial_{r}^{2}\left[r^{d-2 p+1} f^{p}\right] .
$$

This is a total derivative, so varying $f$ does not lead to a useful equation of motion. To find the equations of motion, we have to vary the metric and curvature tensor with respect to arbitrary variations of the metric. The variation of the Riemann curvature tensor reads, denoting $\zeta_{\mu}{ }^{\nu}=\frac{1}{2} g_{\mu \alpha} \delta g^{\alpha \nu}$,

$$
\left.\delta R_{\alpha \beta}{ }^{\gamma \delta}=\frac{1}{2}\left\{\nabla_{[\alpha}, \nabla^{[\gamma}\right\} \zeta_{\beta]}{ }^{\delta]}-\frac{1}{2} \zeta_{[\alpha}{ }^{\epsilon} R_{\beta] \epsilon}{ }^{\gamma \delta}-\frac{1}{2} \zeta_{\epsilon}^{[\gamma} R_{\alpha \beta} \delta\right] \epsilon .
$$

The first term doesn't yield any contribution, because we can partially integrate the covariant derivative in the Lovelock action and because all indices are totally antisymmetrized, the result will vanish because of the Bianchi identity. The second term will contribute and one finds for the metric (2.3) that $\delta R_{\mu \nu}{ }^{\mu \nu}=\left(\zeta_{\mu}{ }^{\mu}+{\zeta_{\nu}}^{\nu}\right) R_{\mu \nu}{ }^{\mu \nu}$ where one does not sum over $\mu$ and $\nu$.

To get the equation of motion, we should in principle work out the variation of the action with respect to $\zeta_{\mu}{ }^{\nu}$. One finds that the variations with respect to $\mu \neq \nu$ vanish identically, the variations with respect to $\zeta_{r}{ }^{r}$ and $\zeta_{t}{ }^{t}$ yield a term proportional to $Q_{3}+2 Q_{4}$, and the variation with respect to $\zeta_{i}^{i}$ yields a term proportional to a linear combination of $Q_{3}+2 Q_{4}$ and $Q_{1}+Q_{2}+Q_{3}+Q_{4}$. By putting the terms proportional to $Q_{3}+2 Q_{4}$ equal to zero, we obtain the equations of motion for $p$-th order Lovelock gravity in $d+1$ dimensions

$$
\left[\sum_{p} \beta_{p}(-1)^{p} \frac{(d-1) !}{(d-2 p) !} r^{d-2 p} f^{p}\right]^{\prime}=0 .
$$

The other equation that one obtains, which is proportional to $Q_{1}+Q_{2}+Q_{3}+Q_{4}$, is a linear combinations of this field equation and its $r$-derivative, and therefore contains no additional information. 
It is convenient to define $\widehat{\lambda}_{p}$ so that

$$
(d-1) \widehat{\lambda}_{p}=\beta_{p}(-1)^{p} \frac{(d-1) !}{(d-2 p) !}
$$

With our conventions for $\beta_{0}$ and $\beta_{1}$ we have that $\widehat{\lambda}_{0}=1$ and $\widehat{\lambda}_{1}=-1$. If we denote

$$
R(r)=\sum_{p} \widehat{\lambda}_{p} r^{d-2 p} f^{p} \quad Q(r)=\frac{\partial R}{\partial f}=\sum_{p} p \widehat{\lambda}_{p} r^{d-2 p} f^{p-1}
$$

we can express the equation of motion (2.9) as

$$
R(r)=K \Rightarrow \sum_{p} \widehat{\lambda}_{p}\left(\frac{f}{r^{2}}\right)^{p}=K / r^{d}
$$

for some constant $K$. Evaluating $R(r)=K$ at the horizon we find that $\widehat{\lambda}_{0}=K / r_{+}^{d}$, which leads to $K=r_{+}^{d}$. Since (2.3) describes an AdS black hole solution we expect $f(r)$ to behave for large $r$ as

$$
f(r)=\alpha r^{2}+\gamma r^{2-d}+\cdots
$$

Combining (2.12) and (2.13) we deduce that

$$
\sum_{p} \hat{\lambda}_{p} \alpha^{p}=0
$$

and

$$
\gamma=\frac{r_{+}^{d}}{\sum_{p} p \widehat{\lambda}_{p} \alpha^{p-1}}
$$

Obviously $\gamma$ is related to the black hole mass, so we need to assume that $\gamma$ is negative. Note that since $r_{+}$is positive, this leads to

$$
\sum_{p} p \widehat{\lambda}_{p} \alpha^{p-1}<0
$$

Interestingly, (2.12) implies that $f / r^{2}$ is monotonic. There cannot be two values of $r$ for which $f / r^{2}$ takes identical values, because then $r_{+}^{d} / r^{d}$ would have to take identical values as well, which implies $r_{1}=r_{2}$. Thus, most real solutions of (2.12) will give rise to acceptable black hole solutions. In fact, as long as $\gamma<0$ and obviously $\alpha>0, \mathrm{f} / \mathrm{r}^{2}$ increases for very large $r$, and therefore, $f / r^{2}$ has to increase everywhere. Since $r_{+}^{d} / r^{d}$ goes to infinity as $r \rightarrow 0, f / r^{2}$ cannot remain bounded as $r \rightarrow 0$. Since it starts of at the value $\alpha$ at $r=\infty$, decreases as we decrease $r$ and cannot remain bounded, it must become zero somewhere and there will be a horizon. So as long as $f / r^{2}$ remains real and $\gamma<0$, this is indeed a black hole type solution. The only subtlety is that a real solution of $f$ might cease to exist at some finite value of $r$.

So defining

$$
P(x)=\sum_{p} \widehat{\lambda}_{p} x^{p},
$$


for a proper black hole solution, $P(x)$ must be monotonously decreasing between 0 and $\alpha$ with $\alpha$ the smallest positive root of $P(x)$. To determine therefore the conditions for a black hole solution to exist we should require that the extrema of $P(x)$, if any, occur outside the region $x \in[0, \alpha]$. In the following section we will carefully analyze these requirements in the context of 3rd order Lovelock gravity.

Let us now move on to the study of metric fluctuations. We will restrict ourselves to fluctuations around the black hole solution (2.3) in the scalar channel, i.e., $\phi=h_{12}$. Other graviton polarizations can be studied in a similar fashion. The form of the perturbed metric is

$$
d s^{2}=-f(r) d t^{2}+\frac{d r^{2}}{f(r)}+r^{2}\left[\sum_{i=1}^{d-1} d x_{i}^{2}+2 \phi\left(t, r, x_{d-1}\right) d x_{1} d x_{2}\right]
$$

Since $\phi$ only depends on the $\left(t, r, x_{d-1}\right)$ directions of spacetime its Fourier transform can be written as

$$
\phi\left(t, r, x_{d-1}\right)=\int \frac{d \omega d q}{(2 \pi)^{2}} \varphi(r) e^{-i \omega t+i q x_{d-1}} \quad k=(\omega, 0,0, \ldots, 0, q)
$$

The equations of motions for $\varphi$ can be found by expanding the Lagrangian (2.2) to second order in the fluctuating field. Alternatively one can substitute the metric (2.18) in the equations of motion and expand to linear order in $\varphi$. The result is

$$
T_{2} \varphi^{\prime \prime}(r)+T_{2}^{\prime} \varphi^{\prime}(r)+T_{0} \varphi(r)=0
$$

where primes indicate differentiation with respect to the variable $\mathrm{r}$ and $T_{2}(r), T_{0}(r)$ are expressed with the help of (2.11) as follows

$$
T_{2}=\frac{d-3}{2} r^{2} f(r)\left(\partial_{r} Q\right), \quad T_{0}=\frac{T_{2}(r)}{a^{2} f(r)^{2}} \omega^{2}-\frac{1}{2} f(r)\left(\partial_{r}^{2} Q\right) q^{2}
$$

Note that we rescaled the time coordinate $t \rightarrow a t$ so as to set the boundary speed of light to unity. One easily checks that if $f(r)$ is of the form given in (2.13) then $\alpha=\frac{1}{a^{2}}$.

It is convenient to make a coordinate transformation from $r$ to $y$ according to $a f(r) \partial_{r} y(r)=1$ and place equation (2.20) in Schrodinger form

$$
-\partial_{y}^{2} \Psi+\left[q^{2} c_{g}^{2}(y)+V_{1}(y)\right] \Psi=\omega^{2} \Psi
$$

Here $\Psi(y)$ is defined as $\Psi=\varphi \frac{T_{2}^{2}}{\sqrt{f}}$ while

$$
\begin{aligned}
c_{g}^{2} & =\frac{a^{2}}{d-3} \frac{f}{r} \frac{\partial_{r}^{2} Q}{\partial_{r} Q} \quad V_{1}(y)=-a^{2} f^{2} h(y)-\frac{1}{a} \sqrt{f} \partial_{y}\left[f^{-2} \partial_{y} \sqrt{f}\right] \\
h(y) & =-\frac{1}{2 a^{2} f} \partial_{y}\left[\frac{1}{f} \frac{\partial_{y} T_{2}}{T_{2}}\right]-\frac{1}{4 a^{2} f^{2}}\left[\frac{\partial_{y} T_{2}}{T_{2}}\right]^{2} .
\end{aligned}
$$

We are now ready to study the full graviton wave function (2.22). Note that $y(r)$ is a monotonically increasing function of $r$ with $y \rightarrow 0$ at the boundary $r \gg r_{+}$and $y \rightarrow-\infty$ at the horizon $r=r_{+}$. Following $[9,10]$ we consider $(2.22)$ in the limit $q \rightarrow \infty$. In this 
case, $q^{2} c_{g}^{2}(y)$ provides the dominant contribution to the potential except for a small region $y>-\frac{1}{q}$. It is therefore reasonable to approximate the potential with $c_{g}^{2}(y)$ for all $y<0$ and replace it with an infinite wall at $y=0$. Consider now the behaviour of $c_{g}^{2}(y)$ in the proximity of the boundary $y=0$. This is easier to analyze in the original variable $r$. In particular,

$$
c_{g}^{2}=1+C \frac{r_{+}^{d}}{r^{d}}+\cdots
$$

with $C$ a function of the Lovelock coefficients $\lambda_{p}$ equal to

$$
C=\frac{1}{\alpha(d-2)(d-3)} \frac{\sum_{p} p((d-2)(d-3)+2 d(p-1)) \widehat{\lambda}_{p} \alpha^{p-1}}{\left(\sum_{p} p \widehat{\lambda}_{p} \alpha^{p-1}\right)^{2}}
$$

To arrive at (2.25) we used eqs $(2.23),(2.15)$ as well as $\alpha=\frac{1}{a^{2}}$.

Observe that when $C$ is positive, $c_{g}^{2}(r)$ attains a maximum value (greater than one) within the bulk geometry. The same is of course true for $c_{g}^{2}$ as a function of the $y$ coordinate. However, the existence of a maximum for $c_{g}^{2}(y)$ implies the existence of metastable states from the point of view of the boundary theory. Moreover, the group velocity of these states can be determined with the WKB approximation to be greater than unity, i.e., the group velocity approaches $c_{g, \max }>1$ at the same time the phase velocity $\frac{\omega}{q}$ tends to $c_{g, \max }[9,10]$. Hence, for values of the Lovelock parameters $\lambda_{p}$ such that $C>0$, the boundary theory violates causality $[9,10]$. Gauge-gravity duality is therefore applicable only when $C$ is negative. From (2.25) this leads to the condition

$$
\sum_{p} p((d-2)(d-3)+2 d(p-1)) \widehat{\lambda}_{p} \alpha^{p-1}<0
$$

for any $p$-th order Lovelock theory of gravity in $d+1$ dimensions.

\section{Black holes of 3rd order Lovelock gravity}

In this section we will analyze asymptotically AdS black hole solutions of 3rd order Lovelock gravity in seven dimensions with flat horizon. In section 3.1. we will apply the results of section 2 to determine the parameter space of these black hole solutions. In section 3.2. we write down the solutions explicitly. For completeness we write here the action of third order Lovelock gravity in $6+1$ dimensions

$$
S=\int d^{7} x \sqrt{-g}\left[\frac{30}{L^{2}}+R+\frac{\lambda_{1} L^{2}}{12} \mathcal{L}_{2}+\frac{\lambda_{2} L^{4}}{72} \mathcal{L}_{3}\right]
$$

Note that $\lambda_{1}$ and $\lambda_{2}$ are related to the Lovelock parameters $\hat{\lambda}$ defined in (2.10) as

$$
\lambda_{1}=\widehat{\lambda}_{2}, \quad \lambda_{2}=-3 \widehat{\lambda}_{3}
$$




\subsection{Existence of black hole solutions}

We start by noting that the action (3.1) admits AdS spacetime as a solution. In the $d=6$ case the AdS radius is related to the parameter $L$ in (3.1) via

$$
L_{\mathrm{AdS}}=a L
$$

where $a$ satisfies

$$
\lambda_{2}=3 a^{2}\left[\lambda_{1}-a^{2}+a^{4}\right]
$$

It is clear that AdS solutions exist for all $\lambda_{1}$ and $a^{2}>0$, since the value of $\lambda_{2}$ can be determined from (3.4). It is not hard to map this parameter space to the $\left(\lambda_{1}, \lambda_{2}\right)$ plane. However it is more convenient to parameterize the solutions using the variables $\lambda_{1}$ and $\alpha=1 / a^{2}$. Consider a black hole which asymptotes to $A d S_{7}$ with the radius $a=1 / \sqrt{\alpha}$. As explained in section 2 such a black hole exists when $P(x)$ defined by $(2.17)$ is a monotonically decreasing function of $x$ between $x=0$ (where $P(0)=1$ ) and $x=\alpha$, where $\alpha$ is the smallest root of $P(x)$. Using $\widehat{\lambda}_{0}=1, \widehat{\lambda}_{1}=-1, \widehat{\lambda}_{2}=\lambda_{1}$ and $\widehat{\lambda}_{3}=-\frac{\lambda_{2}}{3}$, we can write

$$
P(x)=1-x+\lambda_{1} x^{2}-\frac{\lambda_{2}}{3} x^{3}
$$

Let us now fix the value of $\lambda_{1}$ and find a constraint on $\alpha$ which follows from this condition. Suppose $\lambda_{1}>1 / 4$. For $\lambda_{2}<\lambda_{1}^{2}, P(x)$ develops a minimum at

$$
x_{-}=\frac{\lambda_{1}-\sqrt{\lambda_{1}^{2}-\lambda_{2}}}{\lambda_{2}}
$$

and a maximum at

$$
x_{+}=\frac{\lambda_{1}+\sqrt{\lambda_{1}^{2}-\lambda_{2}}}{\lambda_{2}}
$$

The black hole solution exists as long as $P\left(x_{-}\right) \leq 0$. Hence, $\alpha$ is constrained from above by the value of $x=\alpha_{\max }$ such that

$$
P\left(\alpha_{\max }\right)=0, \quad \frac{\partial P}{\partial x}\left(\alpha_{\max }\right)=0
$$

simultaneously. This gives two solutions; the upper solution corresponds to $P\left(x_{+}\right)=0$, while the lower one is what we need,

$$
\alpha_{\max }=\frac{1-\sqrt{1-3 \lambda_{1}}}{\lambda_{1}}
$$

Something interesting happens at $\lambda_{1}=1 / 3$ where $\alpha_{\max }=3$. For $\lambda_{1}>1 / 3$ there is no real solution of eq. (3.8). In this region the existence of the black hole solution is equivalent to the requirement that $P(x)$ is a monotonic function, i.e. $\lambda_{2}>\lambda_{1}^{2}$. With the help of (2.14) this can be restated as

$$
\frac{3 \alpha^{2}-\sqrt{3(4-\alpha) \alpha^{3}}}{2 \alpha^{3}} \leq \lambda_{1} \leq \frac{3 \alpha^{2}+\sqrt{3(4-\alpha) \alpha^{3}}}{2 \alpha^{3}}, \quad \lambda_{1}>\frac{1}{3}
$$




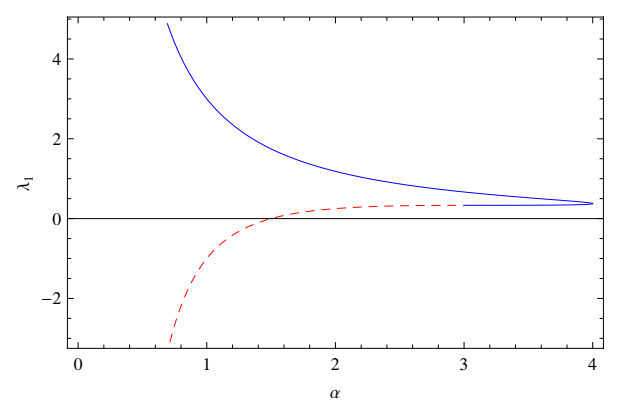

Figure 1. Black holes exist in the region $\alpha \leq \alpha\left(\lambda_{1}\right)$ bounded from the right by the curve. Red dashed part of the curve corresponds to eq. (3.10). Blue solid part of the curve is determined by (3.11). AdS solutions exist for all $\lambda_{1}$ and $\alpha>0$.

The analysis above can be repeated for $0 \leq \lambda_{1} \leq 1 / 4$. Now the value of $\alpha$ is again bounded from above by $\alpha_{\max }$ which is a solution of (3.8). The upper solution with positive $\lambda_{2}$ again corresponds to $P\left(x_{+}\right)=0$, while the lower solution (3.9), now with negative $\lambda_{2}$, is again the upper bound on $\alpha$. The cases of $\lambda_{1}=0$ and $\lambda_{1} \leq 0$ can be analyzed in a similar manner. The result is that black hole solutions exist whenever one of the two conditions, either (3.10), or

$$
0<\alpha<\frac{1-\sqrt{1-3 \lambda_{1}}}{\lambda_{1}}, \quad \lambda_{1}<\frac{1}{3}
$$

is satisfied. Note that the curves defined by (3.10) and (3.11) meet at the point $\left(\lambda_{1}=\right.$ $1 / 3, \alpha=3)$.

The results of this discussion are summarized in figure 1. An AdS solution exists for any values of $\lambda_{1}$ and any positive $\alpha$. The region of the parameter space where black holes exist is bounded by the requirement that $\alpha$ is smaller than the value determined by the curve in figure 1.

\subsection{Explicit solutions}

Explicit black hole solutions for Lovelock gravity have been discussed in many places in the literature. A non exhaustive list of references is [18-26]. To our knowledge however, it has always been assumed that only the real roots of eq. (2.12) describe consistent black holes. Here we will explore other possibilities and find that they indeed satisfy the criteria for valid black hole solutions as described in the previous subsection. In fact, the analysis here can be exactly matched to the previous one although it is the result of independent reasoning.

Following [22] we write the black hole metric in the form

$$
\begin{aligned}
d s^{2} & =-a^{2} f(r) d t^{2}+\frac{d r^{2}}{f(r)}+\frac{r^{2}}{L^{2}} \sum_{i=1}^{5} d x_{i}^{2} \\
f(r) & =\frac{r^{2}}{L^{2}} \frac{\lambda_{1}}{\lambda_{2}} X(r) \\
X(r) & =1+\left(J(r)+\sqrt{J(r)^{2}+G^{3}}\right)^{\frac{1}{3}}+\left(J(r)-\sqrt{J(r)^{2}+G^{3}}\right)^{\frac{1}{3}}
\end{aligned}
$$


where $J(r), G, a$ are defined as

$$
\begin{aligned}
G & =\frac{\lambda_{2}}{\lambda_{1}^{2}}-1 \\
J(r) & =1-\frac{3}{2} \frac{\lambda_{2}}{\lambda_{1}^{2}}+\frac{3}{2} \frac{\lambda_{2}^{2}}{\lambda_{1}^{3}}\left(1-\frac{r_{+}^{6}}{r^{6}}\right) \equiv J_{\infty}-\left(\frac{1}{2}+J_{\infty}+\frac{3}{2} G\right) \frac{r_{+}^{6}}{r^{6}} \\
a^{2} & =\left[\lim _{r \rightarrow \infty} \frac{L^{2}}{r^{2}} f(r)\right]^{-1}=\frac{\lambda_{2}}{\lambda_{1}} X_{\infty}^{-1}
\end{aligned}
$$

and the AdS radius

$$
L_{\mathrm{AdS}}=a L
$$

with $L$ related to the cosmological constant. Notice that $X(r)$ in $L=1$ units is related to the variable $x$ of the previous subsection through $X=\frac{\lambda_{1}}{\lambda_{2}} x$. For our purposes it is useful to express $X(r)$ as

$$
X(r)= \begin{cases}1+\left(J(r)+\sqrt{J(r)^{2}+G^{3}}\right)^{\frac{1}{3}}-\frac{G}{\left(J(r)+\sqrt{J(r)^{2}+G^{3}}\right)^{\frac{1}{3}}} & \lambda_{1}>0 \\ 1+\left(J(r)-\sqrt{J(r)^{2}+G^{3}}\right)^{\frac{1}{3}}-\frac{G}{\left(J(r)-\sqrt{J(r)^{2}+G^{3}}\right)^{\frac{1}{3}}} & \lambda_{1}<0\end{cases}
$$

With $X(r)$ written in this form we see that the solution is determined by the cubic root of the function $M_{ \pm}(r) \equiv \pm \sqrt{J(r)^{2}+G^{3}}+J(r)$, where the \pm sign matches the sign of $\lambda_{1}$. This means that the equations of motion admit three different solutions, classified by the cubic roots of $M_{ \pm}(r)$ left unspecified in (3.12) (and (3.15)). This is also clear from the form of (2.12).

Here we examine which of the three (if not all) and under which conditions, constitute a black hole solution. We will mostly use $X(r)$ as expressed in (3.15) but refer to the one in (3.12) when convenient. Different cases depend on the sign of $\Delta(r) \equiv J^{2}(r)+G^{3}$ sitting under the square root in (3.12). It is useful to consider them separately.

(1) Case I: $G>0 \Leftrightarrow \lambda_{2}>\lambda_{1}^{2}$

Here $\Delta(r) \equiv J^{2}(r)+G^{3}$ is positive for all $r$ which implies $M_{ \pm}(r)= \pm \sqrt{J^{2}(r)+G^{3}}+$ $J(r)$ is real. At the same time $M_{+}(r)$ is positive for all $r$ whereas $M_{-}(r)$ is negative. The three cubic roots of $M_{ \pm}(r)$ are $\left|M_{ \pm}(r)\right|^{\frac{1}{3}} e^{i \frac{\chi_{ \pm}+2 n \pi}{3}}$ with $n=0,1,2$. Naturally, $\chi_{+}=0$ for $M_{+}$and $\chi_{-}=\pi$ for $M_{-}$. Notice that $X(r)$ for all three solutions $(n=0,1,2)$ can be written as

$$
X(r)= \begin{cases}1+\left|M_{+}(r)\right|^{\frac{1}{3}} e^{i \frac{2 n \pi}{3}}-\frac{G}{\left|M_{+}(r)\right|^{\frac{1}{3}}} e^{-i \frac{2 n \pi}{3}} & \lambda_{1} \geq 0 \\ 1+\left|M_{-}(r)\right|^{\frac{1}{3}} e^{i \frac{\pi+2 n \pi}{3}}-\frac{G}{\left|M_{-}(r)\right|^{\frac{1}{3}}} e^{-i \frac{\pi+2 n \pi}{3}} & \lambda_{1}<0\end{cases}
$$

Its imaginary part is equal to

$$
\operatorname{Im} X(r)=\left(\frac{\left|M_{ \pm}(r)\right|^{\frac{2}{3}}+G}{\left|M_{ \pm}(r)\right|^{\frac{1}{3}}}\right) \times \begin{cases}\sin \left(\frac{2 n \pi}{3}\right) & \lambda_{1}>0 \\ \sin \left(\frac{\pi+2 n \pi}{3}\right) & \lambda_{1}<0\end{cases}
$$


When we require the imaginary part to vanish for all $r$ we find that for positive values of $\lambda_{1}$ only the solution with $n=0$ is meaningful. On the other hand, when $\lambda_{1}$ is negative the $n=1$ cubic root is appropriate.

Let us now examine the position of the horizon, i.e., the roots of $X(r)=0$. It is convenient to express $X(r)$ in the form

$$
X(r)=\frac{K(r)+K(r)^{2}-G}{K(r)}
$$

with $K(r)$ the real, same sign, cubic root of $M_{ \pm}(r)$. From eq. (3.18) we see that the solutions of $X(r)=0$ correspond to those of a simple quadratic equation for $K(r)$ in terms of $G$. The discriminant $D=1+4 G$ is always positive when $\lambda_{2}>\lambda_{1}^{2}$ so there are two solutions for $K(r)$

$$
K_{ \pm}=-\frac{1}{2} \pm \frac{1}{2} \sqrt{1+4 G}
$$

Notice that $K_{+}$is positive while $K_{-}$is negative. Since $K(r)$ is either positive or negative (depending on the sign of $\lambda_{1}$ ) for any $r$ when $\lambda_{2}>\lambda_{1}^{2}$, only one of the solutions in (3.19) makes sense in each case. Substituting either expression for $K_{ \pm}$ in terms of $J(r)$ and $G$ into (3.19) and solving for $J(r)$ yields

$$
J=-\frac{1}{2}(1+3 G)
$$

Using then the definition of $J(r)$ from eq. (3.13) one finds that the position of the horizon is $r=r_{+}$.

Next, we determine under which conditions $f(r)$ is positive for $r \geq r_{+}$. It is easy to see that $X(r)$ is positive for $K(r)>K\left(r_{+}\right)$and $\lambda_{1} \geq 0$ while it is negative for negative $\lambda_{1}$ and $K(r)<K\left(r_{+}\right)$. To determine the sign of $X(r)$ outside the horizon it is necessary to understand the monotonicity properties of $K(r)$. Since

$$
\begin{aligned}
\frac{\partial K}{\partial r} & = \begin{cases}\frac{1}{3} K \frac{1}{\sqrt{J(r)^{2}+G^{3}}} \frac{\partial J}{\partial r} & \lambda_{1} \geq 0 \\
-\frac{1}{3} K \frac{1}{\sqrt{J(r)^{2}+G^{3}}} \frac{\partial J}{\partial r} & \lambda_{1}<0\end{cases} \\
\frac{\partial J}{\partial r} & =\frac{3}{2} \frac{\lambda_{2}^{2}}{\lambda_{1}^{3}} \frac{r_{+}}{r^{2}}
\end{aligned}
$$

$K(r)$ is a monotonically increasing function of $r$ when $\lambda_{1}>0$ and monotonically decreasing when $\lambda_{1}<0$. It follows that the sign of $X(r)$ outside the horizon is the same as the sign of $\lambda_{1}$ and given that $\lambda_{2}$ is positive, $f(r)$ is also positive.

In summary, a black hole solution with horizon located at $r=r_{+}$exists for every

$$
\lambda_{2}>\lambda_{1}^{2} \geq 0
$$

(2) Case II: $G=0 \Leftrightarrow \lambda_{2}=\lambda_{1}^{2}$ 
To examine this case it is easier to substitute $\lambda_{2}=\lambda_{1}^{2}$ directly in the equations of motion. Taking the limit $\lambda_{2} \rightarrow \lambda_{1}^{2}$ in (3.12) yields the same result. $X(r)$ is now given by

$$
X(r)=1+[2 \mathcal{J}(r)]^{\frac{1}{3}} \quad \& \quad \mathcal{J}(r)=-\frac{1}{2}+\frac{3}{2} \lambda_{1}\left(1-\frac{r_{+}^{6}}{r^{6}}\right)
$$

It is easy to see that $X(r)$ is real as long as the real cubic root of $\mathcal{J}(r)$ is chosen. Solving the equation $X(r)=0$ for $\mathcal{J}(r)$ determines the position of the horizon to be $r_{+}$again. Positivity of $f(r)$ outside the horizon is guaranteed since $\mathcal{J}(r)$ is a monotonically increasing (decreasing) function of $r$ for $\lambda_{1}$ positive (negative).

Note however that $\frac{\partial X}{\partial r}$ diverges at the point $r_{0}^{6}=\left(\frac{3 \lambda_{1}}{3 \lambda_{1}-1}\right) r_{+}^{6}$ where $\mathcal{J}(r)$ vanishes. The same is true for the scalar curvature of the solution. Nevertheless, if $r_{0}$ is negative or equivalently $0<\lambda<\frac{1}{3}$, the black hole solution remains valid. This is also the case if the divergence occurs behind the horizon, in other words whenever $\lambda_{1}<0$. On the other hand, for $\lambda_{1}>\frac{1}{3}$ and $\lambda_{2}=\lambda_{1}^{2}$ no consistent black hole solution exists. When $\lambda_{2}=\lambda_{1}^{2}=0$ we recover the usual AdS Schwartzchild black hole of Einstein gravity. At the point $\lambda_{2}=\lambda_{1}^{2}=\frac{1}{9}$ the theory degenerates to pure Chern-Simons ${ }^{1}$.

(3) Case III: $G<0 \Leftrightarrow \lambda_{2}<\lambda_{1}^{2}$

In this case $\Delta(r)=J^{2}(r)+G^{3}$ is positive or negative depending on the value of the radial coordinate $r$. To facilitate the analysis we introduce the coordinate $z=\left(\frac{r_{+}}{r}\right)^{6}$ and express $J(z)$ as

$$
J(z)=1-\frac{3}{2} \frac{\lambda_{2}}{\lambda_{1}^{2}}+\frac{3}{2} \frac{\lambda_{2}^{2}}{\lambda_{1}^{3}}(1-z)
$$

The horizon, if it exists, is now located at $z=1 . J(z)$ is a monotonically increasing function of $z$ when $\lambda_{1}$ is negative and decreasing when $\lambda_{1}$ is positive. Notice that under this coordinate transformation eq. $\Delta(z)=0$ is a simple quadratic equation in $z$. In particular, $\Delta(z)$ is positive for $J(z)>\sqrt{-G^{3}}$ and $J(z)<-\sqrt{-G^{3}}$ but negative otherwise.

Let us first consider what happens when $\Delta(z)$ is positive. Clearly, $M_{ \pm}(z)$ is real and its cubic roots can be expressed as $M_{ \pm}(z)=\left|M_{ \pm}(z)\right|^{\frac{1}{3}} e^{i \frac{\chi_{ \pm}+2 n \pi}{3}}$. One can see that both $M_{ \pm}$are positive when $J(z)>\sqrt{-G^{3}}$ and negative when $J(z)<-\sqrt{-G^{3}}$. We then set $\chi \pm=\pi$ for values of $z$ such that $J(z)<-\sqrt{-G^{3}}$ and $\chi_{ \pm}=0$ for $J(z)>\sqrt{-G^{3}}$. Following the same reasoning as in case (I) we find that we must choose $n=0$ for values of $z$ such that $J(z)>\sqrt{-G^{3}}$ and $n=1$ for those satisfying $J(z)<-\sqrt{-G^{3}}$.

On the other hand, when $z$ is such that $-\sqrt{-G^{3}}<J(z)<\sqrt{-G^{3}}$ the function $M_{ \pm}(z)$ is complex and equal ${ }^{2}$ to $M_{ \pm}(z)=J(z) \pm i \sqrt{-J(z)^{2}-G^{3}}$. It can also be written as

\footnotetext{
${ }^{1}$ In fact the symmetries of the theory are enhanced at the points $\lambda_{2}=-\frac{2}{9}+\lambda_{1} \pm \frac{2}{9}\left(1-3 \lambda_{1}\right)^{\frac{3}{2}}$. We thank Jose Edelstein for bringing this to our attention.

${ }^{2}$ Without loss of generality we choose here a positive imaginary part.
} 


$$
\begin{gathered}
M_{ \pm}(z)=|M| e^{ \pm i \phi(z)} \text { with } \phi \in[0, \pi] \text { and }|M|=\sqrt{-G^{3}} \text {. Then } X(z) \text { is } \\
\qquad X(z)=1+|M|^{\frac{1}{3}} e^{i \frac{ \pm \phi+2 n \pi}{3}}-\frac{G}{|M|^{\frac{1}{3}}} e^{-i \frac{ \pm \phi+2 n \pi}{3}}
\end{gathered}
$$

and $\operatorname{Im} X(z)$ vanishes identically for any $n$. As a result, all three solutions are real in this region and

$$
X(z)=1+2 \sqrt{-G} \cos \left(\frac{ \pm \phi+2 n \pi}{3}\right)
$$

Let us summarize. Denote by $\left(z_{+}, z_{-}\right)$the roots of $\Delta(z)=0$

$$
\begin{aligned}
z_{+} & =\frac{2 \lambda_{1}^{3}-3 \lambda_{1} \lambda_{2}+3 \lambda_{2}^{2}+2\left(\lambda_{1}^{2}-\lambda_{2}\right)^{\frac{3}{2}}}{3 \lambda_{2}^{2}} \\
\& \quad z_{-} & =\frac{2 \lambda_{1}^{3}-3 \lambda_{1} \lambda_{2}+3 \lambda_{2}^{2}-2\left(\lambda_{1}^{2}-\lambda_{2}\right)^{\frac{3}{2}} \lambda_{1}^{3}}{3 \lambda_{2}^{2}}
\end{aligned}
$$

It is easy to see that $z_{+}>z_{-}$for all $\lambda_{2}<\lambda_{1}^{2}$. Depending on whether $\lambda_{1}$ is positive or negative $J\left(z_{+}\right)$is equal to the negative or positive value of $\pm \sqrt{-G^{3}}$. Bearing in mind that the monotonicity properties of $J(z)$ also depend on the sign of $\lambda_{1}$ we deduce that

$$
X(z)= \begin{cases}1-\left|M_{+}(z)\right|^{\frac{1}{3}}+\frac{G}{\left|M_{+}(z)\right|^{\frac{1}{3}}} & z>z_{+} \\ 1+2 \sqrt{-G} \cos \left(\frac{\phi+2 n \pi}{3}\right) & z_{-}<z<z_{+} \\ 1+\left|M_{+}(z)\right|^{\frac{1}{3}}-\frac{G}{\left|M_{+}(z)\right|^{\frac{1}{3}}} & z<z_{-}\end{cases}
$$

for positive $\lambda_{1}$ and

$$
X(z)= \begin{cases}1-\left|M_{-}(z)\right|^{\frac{1}{3}}+\frac{G}{\left|M_{-}(z)\right|^{\frac{1}{3}}} & z<z_{-} \\ 1+2 \sqrt{-G} \cos \left(\frac{-\phi+2 n \pi}{3}\right) & z_{-}<z<z_{+} \\ 1+\left|M_{-}(z)\right|^{\frac{1}{3}}-\frac{G}{\left|M_{-}(z)\right|^{\frac{1}{3}}} & z>z_{+}\end{cases}
$$

for $\lambda_{1}$ negative. Notice that it is impossible to construct a black hole solution for $\lambda_{1}^{2}>\lambda_{2}$ which is continuous both at $z=z_{+}$and at $z=z_{-}$. Consider for instance the case of positive $\lambda_{1}$. From the analysis of the imaginary part of $X(z)$ we see that $X^{+}\left(z_{+}\right)=X^{-}\left(z_{+}\right)$requires $n=1$ whereas $X^{+}\left(z_{-}\right)=X^{-}\left(z_{-}\right)$requires $n=0$. The opposite is true when $\lambda_{1}$ is negative. ${ }^{3}$

There are a number of ways to circumvent this problem and built consistent black hole solutions. The obvious one is to constrain the parameter space of $\lambda_{1}, \lambda_{2}$ so that one of the branches in (3.28) and (3.28) is behind the horizon. In other words, we must require that at least one of $z_{ \pm}$is greater than unity. We will now examine this case in detail.

\footnotetext{
${ }^{3}$ Note that the roots $z_{ \pm}$are exactly equal to $P\left(x_{ \pm}\right)$, i.e., the values of $P(x)$ defined in section 2 at the extrema.
} 
Suppose first that $z_{+}>1$ but $0<z_{-}<1$. These two inequalities can be simultaneously solved in the region $\lambda_{1}^{2}>\lambda_{2}$ for either positive or negative $\lambda_{1}$. In the former case, i.e., of positive $\lambda_{1}, X(z)$ is given by

$$
X(z)= \begin{cases}1+2 \sqrt{-G} \cos \left(\frac{\phi}{3}\right) & z_{-}<z<z_{+} \\ 1+\left|M_{+}(z)\right|^{\frac{1}{3}}-\frac{G}{\left|M_{+}(z)\right|^{\frac{1}{3}}} & z<z_{-}\end{cases}
$$

Note that we have chosen $n=0$ for the two branches to smoothly connect outside the horizon. This solution becomes singular at $z=z_{+}$. For $z=1<z_{+}$to define an event horizon, it should be a solution of the eq. $X(z)=0$ with $X(z)$ given by the first branch of (3.28). However, for $\phi \in[0, \pi]$ the first branch is strictly positive for any value of $z$. As a result there is no consistent black hole solution in this region of parameter space.

Things are different when $\lambda_{1}$ is negative. Then $X(z)$ can be written as

$$
X(z)= \begin{cases}1-\left|M_{-}(z)\right|^{\frac{1}{3}}+\frac{G}{\left|M_{-}(z)\right|^{\frac{1}{3}}} & z<z_{-} \\ 1+2 \sqrt{-G} \cos \left(\frac{-\phi+2 \pi}{3}\right) & z_{-}<z<z_{+}\end{cases}
$$

with a singularity at $z_{+}$hidden behind an event horizon at $z=1$. To see that $z=1$ is indeed a solution of the eq. $X(z)=0$ in the "complex" branch of (3.29), bring the eq. $X(z)=0$ in the form

$$
\phi+2 \pi=3 \arccos \left[-\frac{1}{2 \sqrt{-G}}\right]
$$

and take the cosine function on both sides using the identity $\cos 3 x=4 \cos ^{3} x-3 \cos x$. This directly leads to eq. (3.20) which implies $z=1$. Note however that the existence of a horizon in this case requires $\lambda_{2} \leq \frac{3}{4} \lambda_{1}^{2}$. Otherwise, $\sqrt{-G}<\frac{1}{2}$ and $X(z)$ in the "complex" branch is guaranteed to be strictly positive.

To determine the sign of $X(z)$ outside the horizon it is convenient to consider the behavior of the "real branch" (by continuity the same will be true in the "complex" branch). Recall that it can be written as in (3.18) with $K(z)$ the real, same sign, cubic root of $M(z)$. From the previous analysis, we know that $M(z)$ is always negative in this region implying the same for $K(z)$. Moreover, $K^{2}+K-G$ is always positive since for $\lambda_{2} \leq \frac{3}{4} \lambda_{1}^{2}$ the discriminant $D=1+4 G$ is negative. It follows that $X(z)$ outside the horizon is negative. Given that $\lambda_{1}$ is also negative, we deduce that $f(z)$ is positive for all $z<1$. In summary we find that a black hole solution with $X(z)$ given by (3.29) is valid for $\lambda_{1}, \lambda_{2}$ which satisfy

$$
\lambda_{1}<0 \quad \& \quad \lambda_{2}<\lambda_{1}-\frac{2}{9}-\frac{2}{9}\left(1-3 \lambda_{1}\right)^{\frac{3}{2}} \quad \text { OR } \quad \lambda_{1}-\frac{2}{9}+\frac{2}{9}\left(1-3 \lambda_{1}\right)^{\frac{3}{2}}<\lambda_{2}<\frac{3}{4} \lambda_{1}^{2}
$$

Another possible case is for both $z_{ \pm}$to be greater than one. This is actually possible only when $\lambda_{1}$ is negative. Following a similar line of reasoning we can then show that a consistent black hole solution with $X(z)$ equal to

$$
X(z)=1-\left|M_{-}(z)\right|^{\frac{1}{3}}+\frac{G}{\left|M_{-}(z)\right|^{\frac{1}{3}}} \quad z<z_{-},
$$


a singularity at $z=z_{+}$and a horizon for $z=1$ exists as long as

$$
\lambda_{1}<0 \quad \frac{3}{4} \lambda_{1}^{2}<\lambda_{2}<\lambda_{1}^{2}
$$

One might have thought that these possibilities exhaust the spectrum of black hole solutions for $\lambda_{1}^{2}>\lambda_{2}$. This however is not true. Notice that the two distinct solutions of $\Delta(z)=0$ are not necessarily positive. In fact, either or both $z_{ \pm}$can be negative. This implies that the three branches in (3.28) ((3.28)) may be reduced to two or one branch where the continuity ${ }^{4}$ condition $X^{+}\left(z_{ \pm}\right)=X^{-}\left(z_{ \pm}\right)$can be applied.

Consider first the simpler case, where both roots $z_{ \pm}$are negative. Eqs. (3.28) and (3.28) are then reduced to

$$
X(z)=\left\{\begin{array}{ll}
1+\left|M_{-}(z)\right|^{\frac{1}{3}}-\frac{G}{\left|M_{-}(z)\right|^{\frac{1}{3}}} & \lambda_{1}<0 \\
1-\left|M_{+}(z)\right|^{\frac{1}{3}}+\frac{G}{\left|M_{+}(z)\right|^{\frac{1}{3}}} & \lambda_{1}>0
\end{array}\right\}=\frac{K(z)^{2}+K(z)-G}{K(z)}
$$

where $K(z)$ is defined as the real, same sign, cubic root of $M_{ \pm}(z)$. From the analysis of Case I, recall that a necessary condition for the existence of a horizon is $D=1+4 G \geq 0$. Then $K(z)$ evaluated at the horizon is equal to $K(z=1)=-\frac{1}{2}(1-\sqrt{1+4 G})$. Note however that this is incompatible with the first branch of (3.34) since $K(z)$ is positive for all $z$ when $J(z) \geq \sqrt{G^{3}}$. We must then reduce the parameter space region to $\frac{3}{4} \lambda_{1}^{2} \leq \lambda_{2}<\lambda_{1}^{2}$ with $\lambda_{1} \geq 0$ and only consider the second of branch of (3.34). Now $K(z)$ is negative and monotonically increasing with $z$. It follows that $K(z)<K(z=1)$ when $z<1$ so that $K^{2}+K-G<0$ and therefore $X(z)$ carries the same sign with $\lambda_{1}$ outside the horizon. This proves that $f(z)>0$ outside the horizon given that $\lambda_{2}>0$.

Combining the necessary conditions for a black hole solution to exist in this case, i.e., $z_{ \pm}<0$ and $\frac{3}{4} \lambda_{1}^{2} \leq \lambda_{2}<\lambda_{1}^{2}$ and $\lambda_{1}>0$, we find that eq. (3.12) defines a black hole geometry (with the cubic roots taken to be the real ones) as long as

$$
0<\lambda_{1}<\frac{1}{3} \quad \& \quad 0<-\frac{2}{9}+\lambda_{1}+\frac{2}{9}\left(1-3 \lambda_{1}\right)^{\frac{3}{2}}<\lambda_{2}<\lambda_{1}^{2}
$$

Let us move on to the case where $z_{+}$is positive. Recall that we must also specify whether $z_{+}$is greater or smaller than one, i.e., inside or outside the horizon. First let us consider the situation for $0<z_{+} \leq 1$ and $z_{-}<0$. Simultaneously satisfying both inequalities requires $\lambda_{1}$ to be positive. Then (3.28) leads to

$$
X(z)= \begin{cases}1-\left|M_{+}(z)\right|^{\frac{1}{3}}+\frac{G}{\left|M_{+}(z)\right|^{\frac{1}{3}}} & z>z_{+} \\ 1+2 \sqrt{-G} \cos \left(\frac{\phi+2 \pi}{3}\right) & z<z_{+}\end{cases}
$$

The horizon now lies in the "real" branch and $f(z)$ can be shown to be positive in a similar manner as before. Existence of a horizon requires $\frac{3}{4} \lambda_{1}^{2} \leq \lambda_{2}<\lambda_{1}^{2}$. When combined with

\footnotetext{
${ }^{4}$ Imposing $X^{+}\left(z_{+}\right)=X^{-}\left(z_{+}\right)$is not sufficient to ensure continuity of the solution. Nonetheless, it is not difficult to show that first and second derivatives coincide as well.
} 
$0<z_{+} \leq 1$ and $z_{-}<0$ leads to

$$
\begin{aligned}
& 0<\lambda_{1}<\frac{8}{27} \quad \& \quad \quad \frac{3}{4} \lambda_{1}^{2} \leq \lambda_{2}<-\frac{2}{9}+\lambda_{1}+\frac{2}{9}\left(1-3 \lambda_{1}\right)^{\frac{3}{2}} \\
& \frac{8}{27} \leq \lambda_{1}<\frac{1}{3} \quad \& \quad-\frac{2}{9}+\lambda_{1}-\frac{2}{9}\left(1-3 \lambda_{1}\right)^{\frac{3}{2}}<\lambda_{2}<-\frac{2}{9}+\lambda_{1}+\frac{2}{9}\left(1-3 \lambda_{1}\right)^{\frac{3}{2}}
\end{aligned}
$$

Eq. (3.37) represents the necessary and sufficient conditions on the Lovelock parameters for a black hole solution with $X(z)$ given by (3.36) to exist.

On the other hand when $z_{+}>1$ and $z_{-}$is negative, $X(z)$ can be expressed by a single "complex" branch with singularity at $z=z_{+}$hidden behind the horizon

$$
X(r)=1+2 \sqrt{-G} \cos \left(\frac{\phi+2 n \pi}{3}\right) \quad z<z_{+}, \lambda_{1} \neq 0, \phi \in[-\pi, \pi]
$$

Existence of a horizon reduces the parameter space region further to $\lambda_{1}<\frac{3}{4} \lambda_{1}^{2}$. With arguments similar to the ones previously used we can prove that $f(z)$ is positive outside the horizon. We therefore find again a consistent black hole solution. The parameter space region satisfying the above necessary and sufficient conditions is

$$
\begin{array}{cccll}
\lambda_{1}<0 & \& & -\frac{2}{9}+\lambda_{1}-\frac{2}{9}\left(1-3 \lambda_{1}\right)^{\frac{3}{2}}<\lambda_{2}<0 & \text { OR } & 0<\lambda_{2}<-\frac{2}{9}+\lambda_{1}+\frac{2}{9}\left(1-3 \lambda_{1}\right)^{\frac{3}{2}} \\
0<\lambda_{1}<\frac{1}{4} \quad \& \quad-\frac{2}{9}+\lambda_{1}-\frac{2}{9}\left(1-3 \lambda_{1}\right)^{\frac{3}{2}} \leq \lambda_{2}<0 & \text { OR } & 0<\lambda_{2} \leq \frac{3}{4} \lambda_{1}^{2} \frac{1}{4}<\lambda_{1}<\frac{8}{27} \\
-\frac{2}{9}+\lambda_{1}-\frac{2}{9}\left(1-3 \lambda_{1}\right)^{\frac{3}{2}}<\lambda_{2} \leq \frac{3}{4} \lambda_{1}^{2}
\end{array}
$$

It is useful to note that the expressions in (3.38) are not valid when $\lambda_{1}=0$. In fact a black hole solution exists only when $\lambda_{2}$ vanishes as well. Understanding the $\lambda_{2} \rightarrow 0$ limit is also subtle. Computing $X(z)=1+2 \sqrt{-G} \cos \left[\frac{1}{3} \arccos \left[\frac{J(z)}{\sqrt{-G^{3}}}\right]+\frac{2 n \pi}{3}\right]$ for $\lambda_{2}=0$ we find that a solution to $X(z)=0$ exists only for $n=1$. This is in agreement with known results on Gauss-Bonnet gravity; the solution with $n=1$ is continuously connected to the Gauss-Bonnet black hole. For $n=2$ we recover the asymptotically AdS Gauss-Bonnet solution with naked singularity.

\section{Weyl anomaly and the correspondence between flux positivity and causality}

In this section we compute the Weyl anomaly for the third order Lovelock theory in $A d S_{7}$ and determine the values of $t_{2}$ and $t_{4}$ using the results of [14]. We then show that the positivity of energy flux condition precisely matches the causality condition studied in section 2. To compute the anomaly, we generalize the analysis of [14] where the Weyl anomaly was computed for a CFT defined by Gauss-Bonnet gravity in $A d S_{7}$, to the theory defined by (3.1). The description below will be minimal; for details of this procedure the reader is encouraged to consult [14]. The starting point is the ansatz

$$
d s^{2}=L_{\mathrm{AdS}}^{2}\left(\frac{1}{4 \rho^{2}} d \rho^{2}+\frac{g_{i j}}{\rho} d x^{i} d x^{j}\right)
$$


where

$$
g_{i j}=g_{i j}^{(0)}+\rho g_{i j}^{(1)}+\rho^{2} g_{i j}^{(2)}+\mathcal{O}\left(\rho^{3}\right)
$$

is an expansion in powers of the radial coordinate $\rho$. One can now solve the equations of motions derived from (3.1) order by order in the $\rho$ expansion and determine $g_{i j}^{(i)}, i=1, \ldots$ in terms of $g_{i j}^{(0)}$. The resulting expansion (4.2) is then substituted back into $\sqrt{\operatorname{det} g} \mathcal{L}$ and the coefficient of $1 / \rho$ term encodes the anomaly.

As in $[14]$ we take the boundary metric to be of the form

$$
g_{i j} d x^{i} d x^{j}=f\left(x^{3}, x^{4}\right)\left[\left(d x^{1}\right)^{2}+\left(d x^{2}\right)^{2}\right]+\sum_{i=3}^{6}\left(d x^{i}\right)^{2}
$$

and use Mathematica to solve the equations of motion order by order in $\rho$. The leading non-trivial term in the equations of motion relates the value of the AdS radius with the cosmological constant via eq. (3.4). The next to leading term in the equations of motion determines $g^{(1)}$, which is the same as in the Einstein-Hilbert case [27, 28]:

$$
g_{i j}^{(1)}=-\frac{1}{4}\left(R_{i j}-\frac{1}{10} R g_{i j}^{(0)}\right)
$$

The solution for $g_{i j}^{(2)}$ is more non-trivial and involves both $\lambda_{1}$ and $\lambda_{2}$.

Substituting (4.1) together with the solution (4.3) into the action (3.1) and extracting the $1 / \rho$ term in the integrand we obtain an expression of the form $\int d^{6} x \sqrt{\operatorname{det} g^{(0)}} \mathcal{A}_{W}$. We then demand that the coefficient in front of every term in the expression

$$
\mathcal{A}_{W}-\sum_{i=1}^{3} b_{i} I_{i}-\sum_{i=1}^{7} c_{i} C_{i}=0
$$

vanishes. In eq. (4.5) the $I_{i}$ are the B-type anomaly terms composed out of the Weyl tensor, and $C_{i}$ are the total derivative terms. Both can be found in appendix A of [29]. This completely fixes $b_{i}$ and $c_{i}$. Since we are interested in the values of $t_{2}$ and $t_{4}$, it is sufficient to obtain the results for $b_{2} / b_{1}$ and $b_{3} / b_{1}$. These expressions are still too big to be quoted here. As in [14] we can use them, together with the free field theory results, ${ }^{5}$ to determine the values of $t_{2}$ and $t_{4}$ which determine the angular distribution of the energy flux

$$
\langle\mathcal{E}\rangle=\frac{\left\langle\epsilon_{i k}^{*} T_{i k} \mathcal{E}(\widehat{n}) \epsilon_{l j} T_{l j}\right\rangle}{\left\langle\epsilon_{i k}^{*} T_{i k} \epsilon_{l j} T_{l j}\right\rangle}=\frac{q_{0}}{\Omega_{4}}\left[1+t_{2}\left(\frac{\epsilon_{i l}^{*} \epsilon_{l j} n_{i} n_{j}}{\epsilon_{i j}^{*} \epsilon_{i j}}-\frac{1}{5}\right)+t_{4}\left(\frac{\left|\epsilon_{i j} n_{i} n_{j}\right|^{2}}{\epsilon_{i j}^{*} \epsilon_{i j}}-\frac{2}{35}\right)\right]
$$

\footnotetext{
${ }^{5}$ It should be noted that the resulting expressions for $t_{2}$ and $t_{4}$ are of course valid in the strongly interacting CFT defined by Lovelock gravity in $A d S_{7}$. The use of the free theory parametrization for the $b_{i}$ and $\mathcal{A}, \mathcal{B}, \mathcal{C}$ is a technical trick which works because the relation is linear and there are three independent types of free CFTs in six dimension which contain scalar, fermion and antisymmetric two-form fields.
} 
where $\Omega_{4}=\frac{8 \pi^{2}}{3}$. More precisely, we use

$$
\begin{aligned}
1 & =-\left(\frac{28}{3} n_{s}+\frac{896}{3} n_{f}+\frac{8008}{3} n_{a}\right) \\
\frac{b_{2}}{b_{1}} & =\left(\frac{5}{3} n_{s}-32 n_{f}-\frac{2378}{3} n_{a}\right) \\
\frac{b_{3}}{b_{1}} & =\left(2 n_{s}+40 n_{f}+180 n_{a}\right)
\end{aligned}
$$

and eqs (4.9)-(4.11) in [14] to determine $t_{2}$ and $t_{4}$. It turns out that upon the substitution of (3.4), the resulting expressions are remarkably simple:

$$
t_{2}=-10 \frac{2 \lambda_{1}+3 a^{2}\left(a^{2}-1\right)}{\lambda_{1}+a^{2}\left(3 a^{2}-2\right)} \quad t_{4}=0
$$

In section 2 we analyzed the propagation of a graviton with helicity 2 in the black hole background. The absence of metastable states propagating with speed larger than the speed of light gives rise to the constraint $C \leq 0$, or, using the explicit expression for $C(2.25)$,

$$
\frac{5 \lambda_{1}+a^{2}\left(9 a^{2}-8\right)}{\left[\lambda_{1}+a^{2}\left(3 a^{2}-2\right)\right]^{2}} \geq 0
$$

We want to compare (4.9) with the condition coming from the positivity of the energy flux (4.6). The corresponding constraint is given by the first line in eq. (4.8) in [14] which can be written as

$$
5-t_{2} \geq 0
$$

Here we used the fact that $t_{4}$ vanishes in the case of third order Lovelock gravity. Using (4.8) we can write eq. (4.10) as

$$
\frac{5 \lambda_{1}+a^{2}\left(9 a^{2}-8\right)}{\lambda_{1}+a^{2}\left(3 a^{2}-2\right)} \geq 0
$$

Note the remarkable similarity between eqs. (4.9) and (4.11): the numerators in both formulas are exactly the same. To ensure a complete matching between the two results we only need to show that the denominator in eq. (4.11) is always positive for the black hole solutions. Incidentally, there are AdS solutions for which this denominator can be negative. This is because the space of all AdS solutions is parameterized by all possible values of $\lambda_{1}$ and all possible positive values of $a^{2}$, since the value of $\lambda_{2}$ can always be determined from (3.4).

In sections 2 and 3 we analyzed the conditions for black hole solutions to exist. One can show that in the parameter region where the black holes exist (see figure 1) the denominator in (4.11) is indeed always positive. In fact, it vanishes along the dashed red curve in figure 1, which is the border of the parameter space where black hole solutions are allowed. Another way to see this is to note that the denominator in (4.11) is proportional to (minus) the expression (2.16). To verify this, one needs to substitute the expression for $\lambda_{2}(3.4)$ into (2.16). 


\section{Discussion}

In this paper we investigated the relation between causality and positivity of the energy flux for CFTs defined by Lovelock gravities in AdS spacetimes. For a generic Lovelock gravity in arbitrary spacetime dimensions we formulate the condition of existence of asymptotically AdS black holes. We study the propagation of gravitons of helicity 2 in these backgrounds and determine constraints on the Lovelock coefficients resulting from causality of the boundary theory. We then consider cubic Lovelock gravity in $A d S_{7}$ in more detail, and write down the black hole solutions explicitly. To compute the parameters $t_{2}$ and $t_{4}$ in terms of the Lovelock coefficients $\lambda_{1}$ and $\lambda_{2}$ we perform a holographic computation of the Weyl anomaly along the lines of [14]. The coefficients of the B-terms in the anomaly determine the three point functions of the stress energy tensor which are in turn related to the values of $t_{2}$ and $t_{4}$ [14]. We find exact matching between the causality constraint and the corresponding energy flux positivity constraint: for given values of $\lambda_{1}$ and $\lambda_{2}$, as long as the black hole solution exists, causality is preserved whenever the energy flux is positive.

We expect other helicities to match as well (see e.g . [12, 13, 15, 16]). In fact, it would not be surprising if any Lovelock theory of gravity in any dimensions will have the matching property. Our results [see eqs. (2.25), (4.9) and (4.8), and subsequent discussion] lead us to the following conjectured expression

$$
t_{2}-(d-1)=-\frac{d-1}{(d-2)(d-3)} \frac{\sum_{p} p((d-2)(d-3)+2 d(p-1)) \widehat{\lambda}_{p} \alpha^{p-1}}{\sum_{p} p \widehat{\lambda}_{p} \alpha^{p-1}}
$$

for a $p$-th order Lovelock theory in $d+1$ dimensions. We also conjecture that $t_{4}=0$ in all of these theories. Note that eq. (5.1) reproduces both the third order Lovelock gravity result in seven dimensions (4.8) and the Gauss-Bonnet result in any dimensions (eq. (3.32) of [16]; see also eq. (6.35) of [15]) The causality condition (2.26) together with (2.16) then matches precisely the energy flux positivity condition for arbitrary $p$ and $d$.

It is natural to ask how far the correspondence goes, both on the gravity side and on the field theory side. Can it be that all higher derivative gravities share this property? At this stage we do not have a definite answer since the black hole solutions are not known given that the equations of motion are complicated. However one should note that we only need an asymptotic behavior of the black hole metric to probe causality violation. It might be possible to obtain such an asymptotic behavior in a generic higher derivative gravity. It will be harder to establish the condition for the black hole existence: as we have seen, this condition is crucial for the matching to work. Namely, the positivity of energy condition may be naively violated precisely at the point where the black hole solution ceases to exist. Understanding these issues is important if we are to understand better the role higher derivative gravities play in AdS/CFT.

Another question is whether the correspondence between causality at finite temperature and the positivity of energy flux can be shown directly in the field theory. The gravity calculation implies that it is the near boundary behavior of the metric which is responsible for causality violation in the boundary theory. This seems to imply that by studying causality in the short-distance behavior of the two-point function of the stress-energy tensor 
at finite temperature we should be able to constrain the three-point functions. It should be interesting to see whether this can be made precise.

One of the interesting results of this paper is the existence of regions in the parameter space where AdS solutions exist but black hole solutions do not. This is unlike EinsteinHilbert gravity where existence of an AdS solution implies existence of an asymptotically AdS black hole. From the point of view of the AdS/CFT correspondence the HawkingPage transition between the black hole and thermal AdS space [30, 31] can be mapped to the Hagedorn transition in field theory. Indeed, the exponential density of gauge-invariant states $\rho \sim \exp \left(\beta_{H} E\right)$ in large $N$ gauge theories implies that partition function diverges for temperatures $T>1 / \beta_{H}$. Of course this divergence signifies the transition between the phases with $\mathcal{O}\left(N^{0}\right)$ and $\mathcal{O}\left(N^{2}\right)$ degrees of freedom. The black hole is the gravitational description of the latter, high temperature phase. Now the fact the the black holes do not exist for some values of Lovelock parameters may imply that the gravity theory in this region is in some sense dual to a field theory whose density of states does not grow so fast with energy. In fact, higher derivative corrections to the Einstein-Hilbert action are in some cases associated with finite $N$ corrections [32-34], which are expected to smooth out the phase transition.

On the other hand, what happens in Gauss-Bonnet gravity may very well be the case for any Lovelock theory. That is, for those values of the Lovelock parameters for which black holes do not exist, the vacuum AdS solutions may be unstable [35]. It will be interesting to investigate this direction further by studying small fluctuations around the AdS vacuum.

We have obtained the three point functions of the stress energy tensor in a sixdimensional CFT dual to the cubic Lovelock theory. It is interesting that unlike flat space, the cubic Lovelock term contributes to these three point functions. This can be seen from (5.1), which receives contributions from three and two point functions. The third order Lovelock coupling $\widehat{\lambda}_{3}$ enters both explicitly and through the AdS curvature scale $\alpha$. Remarkably, the three point functions of the stress energy tensor satisfy the same relation as the one imposed by supersymmetry, since $t_{4}=0$. Of course this was also the case in Gauss-Bonnet gravity [14], but the vanishing of $t_{4}$ in the $\mathcal{O}\left(R^{3}\right)$ gravity theory is more non-trivial. Again, this poses a question of how special the Lovelock theories are in the AdS/CFT correspondence. Can it be that all these theories are holographically mapped to CFTs which are in some way related to their supersymmetric parents? After all, all known theories whose gravity sector is the Eistein-Hilbert gravity in $A d S_{5}$ are related in some way (e.g. through orbifolding or deformation) to $\mathcal{N}=4$ super Yang-Mills. All these theories indeed retain $a=c$ as a feature.

Finally, let us discuss the implications of our results on the viscosity/entropy bound. It has been shown that inclusion of a Gauss-Bonnet term in gravity violates [9] the $\eta / s \geq 1 / 4 \pi$ bound [36, 37]. Causality implies [9] that there is still a lower bound on $\eta / s$ whose value depends on the dimensionality of the space; the minimal value $\eta / s \geq 1 / 4 \pi \times 219 / 529$ happens for $d=8$ [14]. A straightforward but lengthy computation reveals that the $p$ th order Lovelock term with $p>2$ does not contribute to the viscosity/entropy ratio of the boundary theory. In other words, the value of $\eta / s$ of the dual theory is completely determined by the coefficient of the Gauss-Bonnet term in the gravitational action. After 
arriving at this result we noticed that it has already appeared in the literature [38, 39]. Hence, the viscosity to entropy ratio computed in [9] remains valid for arbitrary Lovelock theories of gravity

$$
\frac{\eta}{s}=\frac{1}{4 \pi}\left(1-\frac{2 d}{d-2} \lambda_{1}\right)
$$

The positivity of energy flux (or, equivalently, causality) constraints place restrictions on the allowed values of $\lambda_{1}$. In the six-dimensional CFT dual to cubic Lovelock theory the constraints are

$$
5-t_{2} \geq 0, \quad 5+\frac{3 t_{2}}{2} \geq 0, \quad 5+3 t_{2} \geq 0
$$

One can determine the physical region of Lovelock parameters by plotting the curves defined by (5.3) and restricting to the physical region in figure 1. It is interesting that the curves defined by inequalities in (5.3) all meet at the point $\left(\alpha=3, \lambda_{1}=1 / 3\right)$. In the physical region (see figure 1) the maximal allowed value of $\lambda_{1}$ comes from the last inequality in (5.3). It is defined by the condition that the function $\lambda_{1}(\alpha)$ defined by $5+3 t_{2}=0$ has a maximum. This happens at $\alpha=15 / 8$ giving rise to $\lambda_{1}=64 / 165$. Clearly, the value of $\eta / s$ is negative for this value of $\lambda_{1}$. It would be interesting to see if the theory develops some pathology before getting to this point.

Note added. After we submitted our paper to the Arxiv we learned of the forthcoming paper by Xian O. Camanho and Jose D. Edelstein [40] which partially overlaps with our results.

\section{Acknowledgments}

We thank J. Edelstein, D. Kutasov, K. Papadodimas, L. Rastelli and M. Rocek for useful discussions. M. K. thanks the YITP at Stony Brook University for hospitality. This work was partly supported by NWO Spinoza grant and the FOM program String Theory and Quantum Gravity.

\section{A The viscosity/entropy ratio of the finite temperature CFT dual to Lovelock gravity.}

Here we compute the viscosity to entropy ratio for any $p$-th order Lovelock theory of gravity in $d+1$ dimensions. Our results agree with those of [38]-[39]. We will mainly follow the discussion in [9]. For this we introduce the definitions and notations below

$$
\begin{aligned}
& z=\frac{r}{r_{+}} \\
& \widetilde{\omega}=\frac{\omega}{r_{+}} \\
& \widetilde{q}=\frac{q}{r_{+}} \\
& \widetilde{f}(z)=\frac{f(z)}{r_{+}^{2}} \\
& \widetilde{R}=\sum_{p} \widehat{\lambda}_{p}\left(\frac{\widetilde{f}}{z^{2}}\right)^{p} z^{d} \\
& \widetilde{Q}=\sum_{p} p \widehat{\lambda}_{p}\left(\frac{\tilde{f}}{z^{2}}\right)^{p-1} z^{d-2} \\
& \widetilde{\gamma}=\frac{\gamma}{r_{+}^{d}}
\end{aligned}
$$


We can now express the equation of motion for $\varphi$ defined in (2.19) with respect to the new variable $z$ as

$$
\left[\widetilde{T}_{2} \partial_{z}^{2} \varphi(z)+\left(\partial_{z} \widetilde{T}_{2}\right) \partial_{z} \varphi(z)+\widetilde{T}_{0} \varphi(z)\right]=0
$$

where

$$
\widetilde{T}_{2}=\frac{d-3}{2} z^{2} \widetilde{f}\left(\partial_{z} \widetilde{Q}\right) \quad \widetilde{T}_{0}=\frac{\widetilde{T}_{2}}{a^{2} \widetilde{f}^{2}} \widetilde{\omega}^{2}-\frac{1}{2} \widetilde{f}\left(\partial_{z}^{2} \widetilde{Q}\right) \widetilde{q}^{2}
$$

and $a^{2}=\frac{1}{\alpha}$ in accordance with the discussion below (2.21). Evaluating the action to quadratic order on the solution of the equation of motion (neglecting contact terms) yields

$$
S=-\left.\frac{1}{2} \frac{a r_{+}^{d}}{l_{p}^{3}} \frac{2}{(d-2)(d-3)} \int \frac{d \omega d q}{(2 \pi)^{2}} T_{2}(z)\left(\partial_{z} \varphi\right) \varphi(z)\right|_{z \rightarrow \infty}
$$

To compute the shear viscosity of the dual theory we set $q=0$ and focus on the low frequency limit of (A.2). Let us first study the behavior of the solution close to the horizon $z=1$. Note that

$$
\left.\frac{T_{0}}{T_{2}}\right|_{q=0}=\frac{\widetilde{\omega}^{2}}{a^{2} \widetilde{f}} \simeq \frac{\widetilde{\omega}^{2}}{d^{2} a^{2}(z-1)^{2}}+\cdots \quad \frac{\partial_{z} T_{2}}{T_{2}} \simeq \frac{1}{z-1}+\cdots
$$

which implies $\varphi \simeq(z-1)^{ \pm \frac{i \widetilde{\omega}}{d a}}$ when $z \simeq 1$. Choosing the infalling boundary condition at the horizon [41-43]-[44] we express the solution of (A.2) in the small frequency limit as

$$
\varphi=J(k)\left(\frac{a^{2} \widetilde{f}}{z^{2}}\right)^{-i \frac{\widetilde{\omega}}{d a}}\left(1-i \frac{\widetilde{\omega}}{d a} g(z)+\cdots\right)
$$

with $g(z)$ regular at $z=1$ and $J(k)$ the boundary source for the field $\varphi$.

It is easy to see that the large $z$ expansion of $g(z)$ is of the form

$$
g(z)=\frac{A}{z^{d}}+\cdots
$$

with $A$ a constant to be determined later. This leads to the following near boundary behavior for $\varphi, \partial_{z} \varphi$ and $\widetilde{T}_{2}(z)$

$$
\begin{aligned}
\varphi(z) & \simeq J(k)\left(1-i \frac{\widetilde{\omega}}{d a} \frac{A+a^{2} \widetilde{\gamma}}{z^{d}}\right) \\
\partial_{z} \varphi & \simeq J(-k) \frac{i \widetilde{\omega}}{a} \frac{A+a^{2} \widetilde{\gamma}}{z^{d+1}} \\
\widetilde{T}_{2}(z) & \simeq \frac{(d-3)(d-2)}{2} \frac{z^{d+1}}{a^{2} \widetilde{\gamma}}
\end{aligned}
$$

Substituting (A.8) into (A.4) yields a boundary term (to leading order in $\widetilde{\omega}$ )

$$
S=-\frac{1}{2} \int \frac{d \omega d q}{(2 \pi)^{2}} \frac{a r_{+}^{d}}{l_{p}^{3}} J(-k) \frac{i \widetilde{\omega}}{a} \frac{A+a^{2} \widetilde{\gamma}}{a^{2} \widetilde{\gamma}} J(k)
$$

The viscosity of the dual plasma is then equal to

$$
\eta=\lim _{\omega \rightarrow 0} \frac{\operatorname{Im} G_{r e t .}(\omega, q=0)}{\omega}=\frac{r_{+}^{d-1}}{l_{p}^{3}} \frac{A+a^{2} \widetilde{\gamma}}{a^{2} \widetilde{\gamma}}
$$


The entropy density of black branes in Lovelock theories of gravity has been discussed in [25]-[26] (see also [45-47]-[48] where the holographic renormalization procedure is employed). Remarkably, it is independent of all Lovelock coefficients $\widehat{\lambda}_{p}$ with $(p>1)$

$$
s=4 \pi \frac{r_{+}^{d-1}}{l_{p}^{3}} .
$$

The ratio of (A.10) to (A.11) then yields

$$
\frac{\eta}{s}=\frac{1}{4 \pi}\left(\frac{A}{a^{2} \widetilde{\gamma}}+1\right)
$$

The contribution from the $p$-th order Lovelock terms is hidden in the ratio $\frac{A}{a^{2} \widetilde{\gamma}}$. Note that the denominator is roughly the product of the black hole mass, $\widetilde{\gamma}$, with the square of the AdS radius factor, $a$. Our objective in the following will be to determine $A$.

Let us start by making the following coordinate transformation

$$
x=\frac{\widetilde{f}}{z^{2}} \quad P(x) \equiv \sum_{p} \widehat{\lambda}_{p} x^{p}=\frac{1}{z^{d}}
$$

Note that the position of the horizon is now at $x=0$ and of the boundary at $x=\alpha$. With these definitions we also have that

$$
\widetilde{Q}=\frac{\partial_{x} P}{P^{1-\frac{2}{d}}} \quad \frac{\partial z}{\partial x}=-\frac{1}{d} \frac{\partial_{x} P}{P^{1+\frac{1}{d}}}
$$

We can therefore express eq. (A.2) in terms of $x, P(x), g(x)$ and expand to linear order in $\widetilde{\omega}$. The result is

$$
H_{2} g^{\prime \prime}(x)+H_{1} g^{\prime}(x)+H=0
$$

where

$$
\begin{aligned}
H_{2} & =x\left(\partial_{x}\right) P\left[(2-d)\left(\partial_{x} P\right)^{2}+d P \partial_{x}^{2} P\right] \\
H & =d\left(\partial_{x}^{2} P\right)\left[\left(\partial_{x} P\right)^{2}-2 P \partial_{x}^{2} P\right]+d P(x) \partial_{x} P \partial_{x}^{3} P \\
H_{1} & =-(d-2)\left(\partial_{x} P\right)^{3}+d x\left(\partial_{x} P\right)^{2} \partial_{x}^{2} P-2 d x P\left(\partial_{x}^{2} P\right)^{2}+d P\left(\partial_{x} P\right)\left(\partial_{x}^{2} P+x \partial_{x}^{3} P\right)
\end{aligned}
$$

Interestingly this equation has a rather simple solution for $\partial_{x} g$

$$
\partial_{x} g=\frac{c_{1}\left(\partial_{x} P\right)^{2}-d P(x)\left(\partial_{x}^{2} P\right)}{x\left[(2-d)\left(\partial_{x} P\right)^{2}+d P(x) \partial_{x}^{2} P\right]} .
$$

Here $c_{1}$ is an integration constant which can be fixed by imposing regularity at the horizon. Observe that in the vicinity of $x=0$ the solution behaves like

$$
\partial_{x} g(x) \simeq \frac{c_{1} \widehat{\lambda}_{1}^{2}-2 d \widehat{\lambda}_{0} \widehat{\lambda}_{2}}{(2-d) \widehat{\lambda}_{1}^{2}+2 d \widehat{\lambda}_{0} \widehat{\lambda}_{2}} \frac{1}{x}+\mathcal{O}\left(x^{0}\right)
$$

Recall that $\widehat{\lambda}_{0}=1, \widehat{\lambda}_{1}=-1$ and $\widehat{\lambda}_{2}=\lambda_{1}$ the coefficient of the Gauss-Bonnet term in the action. Demanding that the solution $g(x)$ be regular at the horizon translates then to

$$
c_{1}=2 d \lambda_{1}
$$


Finally we would like to determine the behavior of $g(x)$ in the vicinity of the boundary $x=\alpha$. In particular, we wish to specify $A$ appearing in eqs (A.10) and (A.12). In other words, we are interested in the asymptotic behavior as a function of the original variable $z$. It is thus convenient to replace $x$ with its near the boundary expression $x \simeq \alpha\left(1+\frac{\widetilde{\gamma}}{z^{d}}\right)$ and expand (A.17) to leading order in $z$ as $z \rightarrow \infty$. From (A.17) we deduce that $\partial_{x} g \simeq$ $c_{2}+\frac{c_{3}}{z^{d}}+\cdots$ with

$$
\begin{array}{r}
\quad=\frac{\sum_{n, m}^{p} n\left(m c_{1}-d(n-1)\right) \widehat{\lambda}_{n} \widehat{\lambda}_{m} \alpha^{n+m-2}}{\sum_{n, m}^{p} n(m(2-d)+d(n-1)) \widehat{\lambda}_{n} \widehat{\lambda}_{m} \alpha^{n+m-1}}=\frac{2 d \lambda_{1}}{(d-2) \alpha} \\
c_{3} \quad=c_{1} a^{2} \widetilde{\gamma}\left[\frac{\sum_{n, m}^{p} n\left(m c_{1}-d(n-1)\right)(n+m-2) \widehat{\lambda}_{n} \widehat{\lambda}_{m} \alpha^{n+m-2}}{\sum_{n, m}^{p} n\left(m c_{1}-d(n-1)\right) \widehat{\lambda}_{n} \widehat{\lambda}_{m} \alpha^{n+m-2}}-\right. \\
\left.-\frac{\sum_{n, m}^{p} n(m(2-d)+d(n-1))(n+m-1) \widehat{\lambda}_{n} \widehat{\lambda}_{m} \alpha^{n+m-1}}{\sum_{n, m}^{p} n(m(2-d)+d(n-1)) \widehat{\lambda}_{n} \widehat{\lambda}_{m} \alpha^{n+m-1}}\right]
\end{array}
$$

where in the last equality of the first line we used (A.19). Note however that

$$
\begin{aligned}
g(z) & =\int d z\left(\partial_{x} g\right)\left(\frac{\partial x}{\partial z}\right) \\
& =\int d z\left(c_{2}+\frac{c_{3}}{z^{d}}+\cdots\right)\left(-d \frac{\widetilde{\gamma}}{z^{d+1}}+\cdots\right) \\
& \simeq \frac{2 d \lambda_{1} a^{2} \widetilde{\gamma}}{(d-2) z^{d}}+\mathcal{O}\left(\frac{1}{z^{2 d}}\right)
\end{aligned}
$$

so the specific value of $c_{3}$ is irrelevant for the leading order boundary asymptotics of $g(z)$. We are now in position to identify $A$ as

$$
A=\frac{2 d \lambda_{1} a^{2} \widetilde{\gamma}}{(d-2)}
$$

and substituting into (A.12) arrive at

$$
\frac{\eta}{s}=\frac{1}{4 \pi}\left(1-\frac{2 d \lambda_{1}}{d-2}\right)
$$

As a result only the Gauss-Bonnet coefficient affects the viscosity to entropy ratio. Any dependence on the black hole mass cancels out [38]-[39].

Open Access. This article is distributed under the terms of the Creative Commons Attribution Noncommercial License which permits any noncommercial use, distribution, and reproduction in any medium, provided the original author(s) and source are credited.

\section{References}

[1] D. Lovelock, The Einstein tensor and its generalizations, J. Math. Phys. 12 (1971) 498 [SPIRES].

[2] B. Zwiebach, Curvature squared terms and string theories, Phys. Lett. B 156 (1985) 315 [SPIRES]. 
[3] B. Zumino, Gravity theories in more than four-dimensions, Phys. Rept. 137 (1986) 109 [SPIRES].

[4] Q. Exirifard and M.M. Sheikh-Jabbari, Lovelock gravity at the crossroads of Palatini and metric formulations, Phys. Lett. B 661 (2008) 158 [arXiv:0705.1879] [SPIRES].

[5] J.M. Maldacena, The large-N limit of superconformal field theories and supergravity, Adv. Theor. Math. Phys. 2 (1998) 231 [Int. J. Theor. Phys. 38 (1999) 1113] [hep-th/9711200] [SPIRES].

[6] E. Witten, Anti-de Sitter space and holography, Adv. Theor. Math. Phys. 2 (1998) 253 [hep-th/9802150] [SPIRES].

[7] S.S. Gubser, I.R. Klebanov and A.M. Polyakov, Gauge theory correlators from non-critical string theory, Phys. Lett. B 428 (1998) 105 [hep-th/9802109] [SPIRES].

[8] S. Nojiri and S.D. Odintsov, On the conformal anomaly from higher derivative gravity in AdS/CFT correspondence, Int. J. Mod. Phys. A 15 (2000) 413 [hep-th/9903033] [SPIRES].

[9] M. Brigante, H. Liu, R.C. Myers, S. Shenker and S. Yaida, Viscosity bound violation in higher derivative gravity, Phys. Rev. D 77 (2008) 126006 [arXiv:0712.0805] [SPIRES].

[10] M. Brigante, H. Liu, R.C. Myers, S. Shenker and S. Yaida, The viscosity bound and causality violation, Phys. Rev. Lett. 100 (2008) 191601 [arXiv:0802.3318] [SPIRES].

[11] D.M. Hofman and J. Maldacena, Conformal collider physics: energy and charge correlations, JHEP 05 (2008) 012 [arXiv:0803.1467] [SPIRES].

[12] A. Buchel and R.C. Myers, Causality of holographic hydrodynamics, JHEP 08 (2009) 016 [arXiv:0906 .2922] [SPIRES].

[13] D.M. Hofman, Higher derivative gravity, causality and positivity of energy in a UV complete QFT, Nucl. Phys. B 823 (2009) 174 [arXiv:0907.1625] [SPIRES].

[14] J. de Boer, M. Kulaxizi and A. Parnachev, AdS $/ C F T_{6}$, Gauss-Bonnet gravity and viscosity bound, JHEP 03 (2010) 087 [arXiv:0910.5347] [SPIRES].

[15] X.O. Camanho and J.D. Edelstein, Causality constraints in AdS/CFT from conformal collider physics and Gauss-Bonnet gravity, JHEP 04 (2010) 007 [arXiv:0911.3160] [SPIRES].

[16] A. Buchel et al., Holographic GB gravity in arbitrary dimensions, JHEP 03 (2010) 111 [arXiv:0911.4257] [SPIRES].

[17] R.R. Metsaev and A.A. Tseytlin, Curvature cubed terms in string theory effective actions, Phys. Lett. B 185 (1987) 52 [SPIRES].

[18] R.C. Myers and J.Z. Simon, Black hole thermodynamics in Lovelock gravity, Phys. Rev. D 38 (1988) 2434 [SPIRES].

[19] J.T. Wheeler, Symmetric solutions to the Gauss-Bonnet extended einstein equations, Nucl. Phys. B 268 (1986) 737 [SPIRES].

[20] M.H. Dehghani and R. Pourhasan, Thermodynamic instability of black holes of third order Lovelock gravity, Phys. Rev. D 79 (2009) 064015 [arXiv:0903.4260] [SPIRES].

[21] M.H. Dehghani, N. Alinejadi and S.H. Hendi, Topological black holes in Lovelock-Born-Infeld gravity, Phys. Rev. D 77 (2008) 104025 [arXiv:0802.2637] [SPIRES].

[22] M.H. Dehghani, N. Bostani and S.H. Hendi, Magnetic branes in third order Lovelock-Born-Infeld gravity, Phys. Rev. D 78 (2008) 064031 [arXiv:0806.1429] [SPIRES]. 
[23] T. Takahashi and J. Soda, Stability of Lovelock black holes under tensor perturbations, Phys. Rev. D 79 (2009) 104025 [arXiv:0902.2921] [SPIRES].

[24] T. Takahashi and J. Soda, Instability of small Lovelock black holes in even-dimensions, Phys. Rev. D 80 (2009) 104021 [arXiv:0907.0556] [SPIRES].

[25] R.-G. Cai, Gauss-Bonnet black holes in AdS spaces, Phys. Rev. D 65 (2002) 084014 [hep-th/0109133] [SPIRES].

[26] R.-G. Cai, L.-M. Cao, Y.-P. Hu and S.P. Kim, Generalized Vaidya spacetime in Lovelock gravity and thermodynamics on apparent horizon, Phys. Rev. D 78 (2008) 124012 [arXiv:0810.2610] [SPIRES].

[27] M. Henningson and K. Skenderis, The holographic Weyl anomaly, JHEP 07 (1998) 023 [hep-th/9806087] [SPIRES].

[28] M. Henningson and K. Skenderis, Holography and the Weyl anomaly, Fortsch. Phys. 48 (2000) 125 [hep-th/9812032] [SPIRES].

[29] F. Bastianelli, S. Frolov and A.A. Tseytlin, Conformal anomaly of $(2,0)$ tensor multiplet in six dimensions and AdS/CFT correspondence, JHEP 02 (2000) 013 [hep-th/0001041] [SPIRES].

[30] E. Witten, Anti-de Sitter space, thermal phase transition and confinement in gauge theories, Adv. Theor. Math. Phys. 2 (1998) 505 [hep-th/9803131] [SPIRES].

[31] S.W. Hawking and D.N. Page, Thermodynamics of black holes in Anti-de Sitter space, Commun. Math. Phys. 87 (1983) 577 [SPIRES].

[32] O. Aharony, A. Fayyazuddin and J.M. Maldacena, The large- $N$ limit of $N=2,1$ field theories from three-branes in F-theory, JHEP 07 (1998) 013 [hep-th/9806159] [SPIRES].

[33] A. Fayyazuddin and M. Spalinski, Large- $N$ superconformal gauge theories and supergravity orientifolds, Nucl. Phys. B 535 (1998) 219 [hep-th/9805096] [SPIRES].

[34] Y. Kats and P. Petrov, Effect of curvature squared corrections in AdS on the viscosity of the dual gauge theory, JHEP 01 (2009) 044 [arXiv:0712.0743] [SPIRES].

[35] D.G. Boulware and S. Deser, String generated gravity models, Phys. Rev. Lett. 55 (1985) 2656 [SPIRES].

[36] P. Kovtun, D.T. Son and A.O. Starinets, Viscosity in strongly interacting quantum field theories from black hole physics, Phys. Rev. Lett. 94 (2005) 111601 [hep-th/0405231] [SPIRES].

[37] Holography and hydrodynamics: diffusion on stretched horizons, JHEP 10 (2003) 064 [hep-th/0309213] [SPIRES].

[38] R. Brustein and A.J.M. Medved, The ratio of shear viscosity to entropy density in generalized theories of gravity, Phys. Rev. D 79 (2009) 021901 [arXiv:0808.3498] [SPIRES].

[39] F.-W. Shu, The quantum viscosity bound in Lovelock gravity, Phys. Lett. B 685 (2010) 325 [arXiv: 0910.0607] [SPIRES].

[40] X.O. Camanho and J.D. Edelstein, Causality in AdS/CFT and Lovelock theory, to appear.

[41] D.T. Son and A.O. Starinets, Minkowski-space correlators in AdS/CFT correspondence: Recipe and applications, JHEP 09 (2002) 042 [hep-th/0205051] [SPIRES].

[42] C.P. Herzog and D.T. Son, Schwinger-Keldysh propagators from AdS/CFT correspondence, JHEP 03 (2003) 046 [hep-th/0212072] [SPIRES]. 
[43] D. Marolf, States and boundary terms: subtleties of lorentzian AdS/CFT, JHEP 05 (2005) 042 [hep-th/0412032] [SPIRES].

[44] K. Skenderis and B.C. van Rees, Real-time gauge/gravity duality, Phys. Rev. Lett. 101 (2008) 081601 [arXiv:0805. 0150] [SPIRES].

[45] G. Kofinas and R. Olea, Vacuum energy in Einstein-Gauss-Bonnet AdS gravity, Phys. Rev. D 74 (2006) 084035 [hep-th/0606253] [SPIRES].

[46] O. Mišković and R. Olea, Counterterms in dimensionally continued AdS gravity, JHEP 10 (2007) 028 [arXiv:0706.4460] [SPIRES].

[47] G. Kofinas and R. Olea, Universal regularization prescription for Lovelock AdS gravity, JHEP 11 (2007) 069 [arXiv:0708.0782] [SPIRES].

[48] G. Kofinas and R. Olea, Universal kounterterms in Lovelock AdS gravity, Fortsch. Phys. 56 (2008) 957 [arXiv:0806.1197] [SPIRES]. 